\title{
Phase-resolved X-ray spectra of magnetars and the coronal outflow model
}

\author{
Romain Hascoët ${ }^{1}$, Andrei M. Beloborodov \\ Physics Department and Columbia Astrophysics Laboratory, Columbia University, 538 West \\ 120th Street New York, NY 10027 \\ Peter R. den Hartog \\ Stanford University HEPL/KIPAC, 452 Lomita Mall, Stanford, CA 94305-4085
}

\begin{abstract}
We test a model recently proposed for the persistent hard X-ray emission from magnetars. In the model, hard X-rays are produced by a decelerating electron-positron flow in the closed magnetosphere. The flow decelerates as it radiates its energy away via resonant scattering of soft X-rays, then it reaches the top of the magnetic loop and annihilates there. We test the model against observations of three magnetars: $4 \mathrm{U}$ 0142+61, 1RXS J1708-4009, and 1E 1841-045. We find that the model successfully fits the observed phase-resolved spectra. We derive constraints on the angle between the rotational and magnetic axes of the neutron star, the object inclination to the line of sight, and the size of the active twisted region filled with the plasma flow. Using the fit of the hard X-ray component of the magnetar spectrum, we revisit the remaining soft X-ray component. We find that it can be explained by a modified two-temperature blackbody model. The hotter blackbody is consistent with a hot spot covering 1-10\% of the neutron star surface. Such a hot spot is expected at the base of the magnetospheric $e^{ \pm}$outflow, as some particles created in the $e^{ \pm}$discharge flow back and bombard the stellar surface.
\end{abstract}

Subject headings: magnetars: individual (1E 1841-045, 1RXS J1708-4009, 4U 0142+61)

- X-rays: stars - plasmas - stars: magnetic field - stars: neutron

\section{Introduction}

Magnetars are isolated neutron stars whose emission is thought to be powered by the decay of intense magnetic fields (Duncan \& Thompson 1992; Thompson \& Duncan 1996). Their persistent X-ray spectrum shows two peaks, near $1 \mathrm{keV}$ and above $100 \mathrm{keV}$ (e.g. Kuiper et al. 2006;

\footnotetext{
${ }^{1}$ hascoet@astro. columbia.edu
} 
Enoto et al. 2010). The soft X-ray component likely comes from the neutron star surface. Its spectrum is modified from a simple Planck shape by the radiative transfer in the atmosphere and magnetosphere of the star, and it has a soft tail extending to $\sim 10 \mathrm{keV}$ where the hard component takes over. The hard X-rays must be produced in the magnetosphere of the neutron star.

For a few magnetars, phase-resolved spectra were measured in the hard X-ray band (den Hartog et al. 2008a,b). They showed bizarre variations with rotational phase. Recently, Beloborodov $(2013 \mathrm{~b})$ proposed a model of decelerating $e^{ \pm}$outflow that makes specific predictions for the hard $\mathrm{X}$-ray spectrum and its variation with rotational phase. In the present paper, we test the model against observations of three magnetars, 1E 1841-045, 4U 0142+61 and 1RXS J1708-4009 (all discovered as anomalous X-ray pulsars). The model is briefly described in Section 2, and the results of data analysis are presented in Section 3. We find that the model successfully fits the data and discuss implications of our results in Section 4 .

\section{Coronal outflow model}

In the model, the hard X-ray emission is powered by the $e^{ \pm}$discharge near the star. The discharge voltage exceeds $10^{9} \mathrm{~V}$ and the initial Lorentz factor of created $e^{ \pm}$exceeds $10^{3}$ (Beloborodov \& Thompson 2007). As the $e^{ \pm}$pairs flow out along the magnetic field lines and fill the extended magnetic loop, they decelerate via resonant scattering of thermal X-rays. Beloborodov (2013b) showed that the outflow has two zones, adiabatic and radiative. The adiabatic zone is near the star where magnetic field $B>10^{13} \mathrm{G}$; in this zone the resonantly scattered photons quickly convert to pairs, increasing the $e^{ \pm}$multiplicity of the flow to $\mathcal{M} \sim 10^{2}$. The radiative zone is at higher altitudes where $B<10^{13} \mathrm{G}$; here the resonantly scattered photons escape and the outflow enters the radiative regime, losing its kinetic energy.

The interaction of radiation and $e^{ \pm}$outflow was studied in detail in Beloborodov (2013a). It was shown that the outflow Lorentz factor $\gamma_{ \pm}$decreases proportionally to the local magnetic field,

$$
\gamma_{ \pm} \approx 10^{2} \frac{B}{B_{Q}}
$$

The average energy of resonantly scattered photons is given by

$$
E_{\mathrm{sc}} \approx \gamma_{ \pm} \frac{B}{B_{Q}} m_{e} c^{2} \approx 5 \gamma_{ \pm}^{2} \mathrm{keV}
$$

Equations (11) and (2) imply that the spectrum of escaping hard X-rays has an average photon index $\Gamma=1.5$ and its luminosity peaks at the high-energy end (Beloborodov 2013b). The spectrum cuts off in the $\mathrm{MeV}$ band, because the multi-MeV photons are emitted in the adiabatic zone and cannot escape. The predicted spectral shape, however, strongly depends on the viewing angle, and may significantly differ from the average spectrum.

For a given position of the observer and location of the active magnetic loop the model predicts a well-defined phase-dependent radiation spectrum that can be quantitatively compared with 
observations. The spectrum can be calculated using the Monte-Carlo technique as described in (Beloborodov 2013b). We have developed a different, independent code that performs a similar calculation using direct integration of the angle-dependent emissivity over the j-bundle. This method does not include photon splitting, which is important at high energies. We have tested the code against the Monte-Carlo results presented in Beloborodov (2013b) and found excellent agreement at photon energies below $400 \mathrm{keV}$.

In this paper, we assume that the magnetosphere is axisymmetric and close to the dipole configuration in the region of interest (where $B<10^{13} \mathrm{G}$ ). The dipole moment of the neutron star $\mu_{\mathrm{m}}$ is provided by measurements of its spindown rate; it is taken from Dib et al. (2007) for $4 \mathrm{U}$ 0142+61, and from Dib et al. (2008) for 1RXS J1708-4009 and 1E 1841-045. We also assume that the active, current-carrying region of the magnetosphere ("j-bundle"), which is filled with the $e^{ \pm}$flow, is axisymmetric. Then the model is completely defined by four remaining parameters:

- $\theta_{j}$, the half opening angle (from the magnetic axis) of the j-bundle footprint on the star.

- $L_{j}$, the bolometric luminosity radiated by the j-bundle.

- $\alpha_{\mathrm{mag}}$, the angle between the rotation axis and magnetic axis of the neutron star.

- $\beta_{\text {obs }}$, the angle between the rotation axis and the observer line of sight.

In addition, the point of zero rotational phase must be kept as a free parameter to fit phase-resolved data. The parameter $L_{j}$ is related to two theoretical quantities - the discharge voltage $\Phi$ and the twist angle of the magnetic field lines $\psi$ (Beloborodov 2009),

$$
L_{j} \simeq 2 \times 10^{35} \psi\left(\frac{\Phi}{10^{10 \mathrm{~V}}}\right)\left(\frac{\mu_{\mathrm{m}}}{10^{32} \mathrm{G} \mathrm{cm}^{3}}\right)\left(\frac{R}{10 \mathrm{~km}}\right)\left(\frac{\theta_{j}}{0.3}\right)^{4} \mathrm{erg} \mathrm{s}^{-1},
$$

where $R$ is the radius of the neutron star, which we fix at $10 \mathrm{~km}$. A twist angle $\psi \sim 1$ is expected for a strongly twisted magnetosphere; measured values of $L_{j}$ and $\theta_{j}$ can provide an order-of-magnitude for the discharge voltage $\Phi$.

The j-bundle footprint can form a hot spot on the neutron star, as some of the relativistic particles created in the $e^{ \pm}$discharge flow back to the stellar surface and bombard it. This motivates us to consider a two-temperature model for the soft X-ray emission from the stellar surface, with the hotter blackbody associated with the j-bundle footprint. The expected area of the hot spot is related to $\theta_{j}$ by

$$
\mathcal{A}_{\mathrm{h}} \sim \frac{1}{4} \theta_{j}^{2} \mathcal{A}_{\mathrm{ns}} \approx 0.02\left(\frac{\theta_{j}}{0.3}\right)^{2} \mathcal{A}_{\mathrm{ns}}
$$

where $\mathcal{A}_{\mathrm{ns}}=4 \pi R^{2}$ is the surface area of the neutron star. The model is illustrated in Figure 1. 


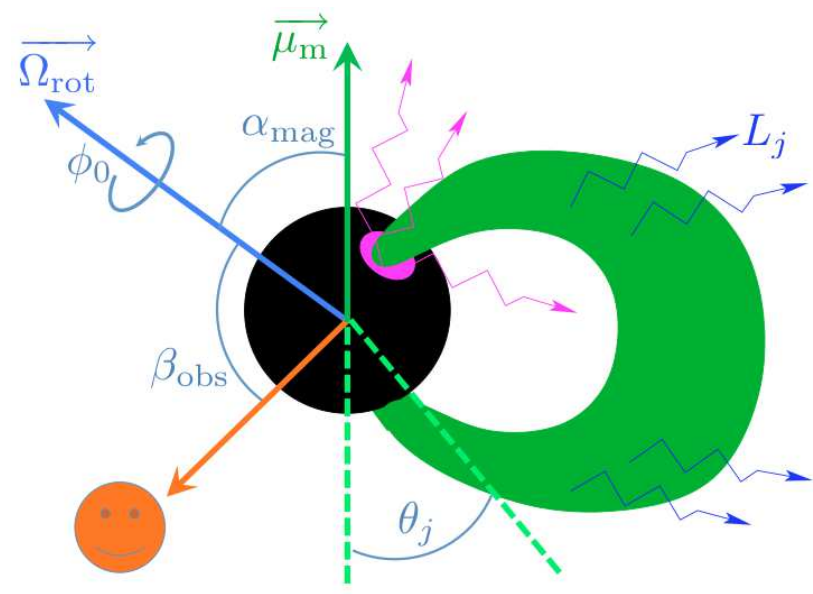

Fig. 1.- Schematic picture of the coronal outflow model indicating its parameters (see text). The current-carrying magnetic loop ("j-bundle", shown in green) is filled by the relativistic $e^{ \pm}$flow launched from the discharge zone near the neutron star. At high altitudes where $B<10^{13} \mathrm{G}$, the $e^{ \pm}$outflow converts its kinetic energy to hard X-rays (blue arrows) via resonant scattering of thermal X-rays. Some particles from the $e^{ \pm}$discharge flow toward the star and bombard it, forming a hot spot at the footprint of the j-bundle (magenta).

\section{Spectral fits}

For 4U 0142+61 and 1RXS J1708-4009, we used the multi-year data collected by INTEGRALISGRI (20-300 keV), INTEGRAL-SPI (10-1000 keV), XMM-Newton (0.55-11.5 keV), ASCA-GIS (0.7 - $12 \mathrm{keV}$, only for 4U 0142+61), RXTE-PCA $(2-60 \mathrm{keV})$ and CGRO-Comptel (upper-limits in the range $0.75-30 \mathrm{MeV}$, see Kuiper et al. 2006, den Hartog et al. 2006). The data set for the two magnetars is the same as in den Hartog et al. (2008b, a); it is described in detail there. The data and fit by the outflow model for 1E 1841-045 are presented in An et al. (2013); below we show the results of An et al. (2013) for comparison with 4U 0142+61 and 1RXS J1708-4009.

\subsection{Hard component}

We first focus on the data above $10 \mathrm{keV}$, where the relativistic outflow dominates the observed emission. In this energy range, we search for a combined fit of the phase-averaged spectrum of the total (pulsed + unpulsed) emission and three phase-resolved spectra of the pulsed emission. The three phase bins are the same as in den Hartog et al. (2008b,a).

The entire parameter space of the model (Section 2) was discretized on a grid, and we calculated $\chi^{2}$ and the corresponding $p$-value for the model at each grid point. The obtained map of $\chi^{2}$ provided a reliable way to find the best fit, avoiding the risk of converging to a local minimum with an 
$p$-value scale

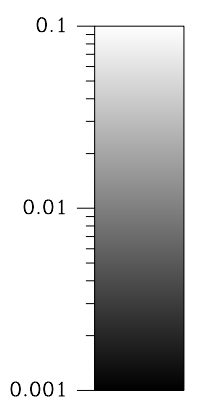

1E $1841-045$

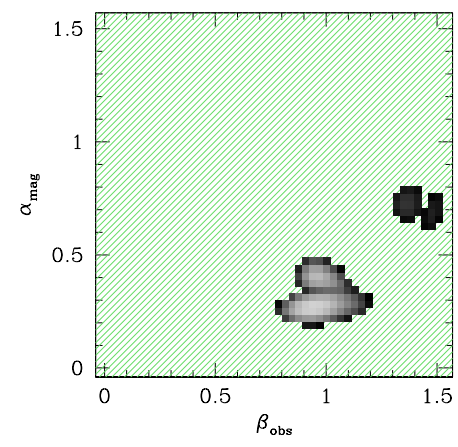

$4 \mathrm{U} 0142+61$

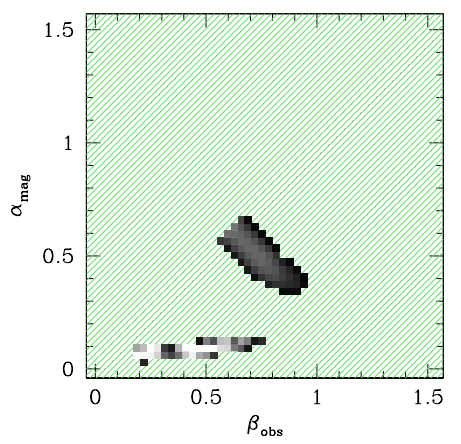

1RXS J1708-4009

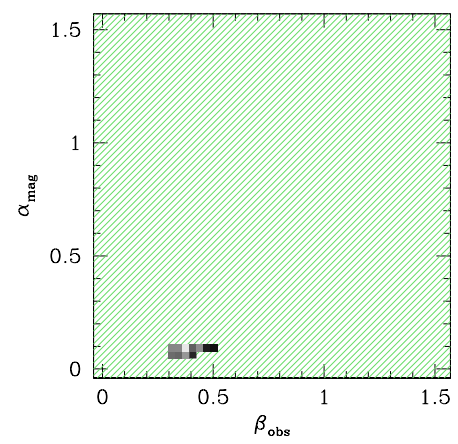

Fig. 2.- Map of $p$-value for the fit by the coronal outflow model for 1E 1841-045 (left), 4U 0142+61 (middle) and 1RXS J1708-4009 (right). The hatched green region has p-values smaller than 0.001.

optimization algorithm. We found that $\chi^{2}$ is a rather complex function of the model parameters and standard optimization software such as XSPEC would fail to identify the best fit. Remarkably, we found that $\chi^{2}$ has a sharp minimum in a well localized region of the parameter space. The best fit parameters for each magnetar are summarized in Table 1.

Figure 2 shows the $p$-value maps on the plane of $\alpha_{\text {mag }}, \beta_{\text {obs }}$ after maximizing the $p$-value (minimizing $\chi^{2}$ ) over the parameters $\theta_{j}$ and $L_{j}$ for each $\alpha_{\text {mag }}, \beta_{\text {obs }}$. One can see that the model completely fails $(p<0.001)$ almost everywhere; this reflects the fact the model is quite "rigid," lacking flexibility in adjusting $\chi^{2}$ to a desired value. Nevertheless, a good fit with $p>0.05$ is obtained in a small region of the parameter space. We conclude that the model (a) successfully fits the data and (b) the fit imposes strong constraints on the parameters.

We note, however, that there is one significant source of degeneracy in the axisymmetric model. One can show that interchanging the values of $\alpha_{\text {mag }}$ and $\beta_{\text {obs }}$ does not change the predicted spectrum. Thus, Figure 2 must be complemented with a similar map obtained by mirror reflection about the line of $\beta_{\mathrm{obs}}=\alpha_{\mathrm{mag}}$. The fact that one of the two angles is found to be small for $4 \mathrm{U}$ 0142+61 and 1RXS J1708-4009 may help to break this degeneracy. Since the line of sight is random, there is no reason for $\beta_{\text {obs }}$ to be small. It is more likely that it is $\alpha_{\text {mag }}$ that is small, as this may be a result of the magnetic field evolution in the neutron star at its birth or later times.

We also explored the model with unfrozen parameter $\mu_{\mathrm{m}}$ and investigated if fitting by this more flexible model allows one to obtain constraints on $\mu_{\mathrm{m}}$, which would be independent from the spindown measurements. We found that such constraints are weak - only a lower limit on the magnetic moment can be derived this way, which is about one order of magnitude below the value inferred from the spindown rate. 
Table 1: Parameters of the best-fit model for the hard X-ray component

\begin{tabular}{ccccccc}
\hline \hline & $\begin{array}{c}\alpha_{\mathrm{mag}} \\
(\mathrm{rad})\end{array}$ & $\begin{array}{c}\beta_{\text {obs }} \\
(\mathrm{rad})\end{array}$ & $\begin{array}{c}\theta_{j} \\
(\mathrm{rad})\end{array}$ & $\begin{array}{c}L_{j}{ }^{\mathrm{a}} @ D_{0}{ }^{\mathrm{b}} \\
\mu_{\mathrm{m}}{ }^{\mathrm{c}}\end{array}$ & $\begin{array}{c}\chi^{2} / \mathrm{dof} \\
\left(10^{32} \mathrm{G} \mathrm{cm}^{3}\right)\end{array}$ \\
\hline 4U 0142+61 & $<0.15$ & $0.5(3)$ & $<0.23$ & $3.9(24) @ 3.6$ & 1.3 & $46 / 41$ \\
1RXS J1708-4009 & $<0.15$ & $0.4(2)$ & $<0.15$ & $3.5(18) @ 3.8$ & 4.6 & $49 / 37$ \\
1E 1841-045 - sol. 1 & $0.3(2)$ & $0.9(2)$ & $<0.4$ & $48(16) @ 8.5$ & 6.9 & $302 / 267$ \\
1E 1841-045 - sol. 2 & $0.7(2)$ & $1.4(1)$ & $<0.4$ & $48(16) @ 8.5$ & 6.9 & $326 / 267$ \\
\hline
\end{tabular}

Note. Uncertainties are given at the $3 \sigma$ level. There are two solutions within $3 \sigma$ for $1 \mathrm{E} 1841-045$. ${ }^{\mathrm{a}}$ In units of $10^{35}\left(D / D_{0}\right)^{2} \mathrm{erg} \mathrm{s}^{-1}$.

${ }^{\mathrm{b}}$ Distance to the object (in $\mathrm{kpc}$ ) estimated from reddening and X-ray extinction along the line of sight (for 4U 0142+61 and 1RXS J1708-4009, Durant \& van Kerkwiik 2006) or from the Galactic rotation of the associated supernova remnant (for 1E 1841-045, Tian \& Leahv 2008).

${ }^{\mathrm{c}}$ Magnetic dipole moment of the neutron star inferred from its spindown rate (see Dib et al. 2007 for $4 \mathrm{U} 0142+61$, Dib et al. 2008 for 1RXS J1708-4009 and 1E 1841-045).

\subsection{Soft component}

Once the hard X-ray component is fitted by the $e^{ \pm}$outflow model, we turn to the remaining soft component below $10 \mathrm{keV}$. Note that the outflow makes a non-negligible contribution below $10 \mathrm{keV}$ (the two components overlap in this region). Thus, understanding the origin of the hard $\mathrm{X}$-ray component and its low-energy extension is important for the correct interpretation of soft X-ray emission. Previous models for the $1-10 \mathrm{keV}$ spectrum involved radiative transfer in the the neutron star atmosphere (e.g. Ho \& Lai 2001; Özel 2001) or resonant scattering by mildly relativistic electrons flowing in the magnetosphere (Thompson et al. 2002). Alternatively, the soft X-ray tail was proposed to be the signature of a hot spot on the star (e.g. Gotthelf \& Halpern 2007).

Motivated by the j-bundle picture described in Section 2, we investigated models that include the possible presence of a hot spot. Two-temperature blackbody (2BB) is a simplest model of this type. The lower temperature is typically associated with a large area (comparable to the area of the neutron star surface) and the higher temperature is associated with the footprint of the j-bundle. This simple model was sufficient to provide a good fit for the spectrum of 1E 1841-045 (An et al. 2013). We found that the 2BB model is insufficient for the soft component of 4U $0142+61$ and 1RXS J1708-4009. This may be expected, as the soft X-ray spectrum should be modified by resonant scattering in the magnetosphere (e.g. Thompson et al. 2002; Rea et al. 2008), although a consistent model for this effect is yet to be developed (see Beloborodov 2013b for discussion). We chose a phenomenological modification of the 2BB model: we replaced the Wien tail of the hotter blackbody by a power law that is smoothly connected to the Planck spectrum at some energy $E_{\text {tail }}$. Smooth connection here means the continuity of the photon spectrum and its first derivative; then $E_{\text {tail }}$ is the only required additional parameter. The temperatures $T_{1}$ and $T_{2}$ of the cold and hot blackbodies, their luminosities $L_{1}$ and $L_{2}$, and $E_{\text {tail }}$ uniquely define our modified 2BB model. 
Table 2: Parameters of the best-fit model for the soft X-ray component

\begin{tabular}{cccccccc}
\hline \hline & $\begin{array}{c}N_{\mathrm{H}} \\
\left(10^{22} \mathrm{~cm}^{-2}\right)\end{array}$ & $\begin{array}{c}k T_{1} \\
(\mathrm{keV})\end{array}$ & $\begin{array}{c}k T_{2} \\
(\mathrm{keV})\end{array}$ & $\begin{array}{c}E_{\text {tail }} \\
(\mathrm{keV})\end{array}$ & $L_{1}{ }^{\mathrm{a}}$ & $L_{2}{ }^{\mathrm{a}}$ & $\chi^{2} / \mathrm{dof}$ \\
\hline 4U 0142+61 & $0.577(5)$ & $0.307(4)$ & $0.602(9)$ & $3.82(6)$ & $2.63(3)$ & $1.36(9)$ & $559 / 448$ \\
1RXS J1708-4009 & $0.99(1)$ & $0.39(1)$ & $0.87(5)$ & $4.0(2)$ & $0.69(3)$ & $0.56(12)$ & $297 / 228$ \\
1E 1841-045 & $2.03(4)$ & $0.45(1)$ & $0.90(4)$ & $\ldots$ & $2.15(7)$ & $0.65(9)$ & $2298 / 2272$
\end{tabular}

Note. ${ }^{a}$ In units of $10^{35}\left(D / D_{0}\right)^{2} \mathrm{erg} \mathrm{s}^{-1}$. See Table 1 for the values of $D_{0}$.

We found that the spectra of 4U 0142+61 and 1RXS J1708-4009 are reasonably well fitted by the modified 2BB model; the results are summarized in Table 2. The formal $\chi^{2}$ is unsatisfying, because of the high spectral resolution of XMM and the inability of simple phenomenological models to describe spectral lines. However the continuum of observed spectra is well reproduced. The residuals are largest below $3 \mathrm{keV}$ and suggest the presence of line-like features; this issue was already pointed by den Hartog et al. (2008a). The best fits of the soft and hard components are shown together in Figure 3 .

For comparison, we also tried to fit the soft component with the sum of one (modified) blackbody and a power law. This model provided acceptable fits for 1RXS J1708-4009 and 1E 1841-045, but not for $4 \mathrm{U} 0142+61\left(\chi^{2} / \mathrm{dof}=774 / 449\right)$. An additional, well known drawback of this model is the large contribution of the power-law below $\sim 1 \mathrm{keV}$, which results in an unrealistically large best-fit value of the hydrogen column density toward the object.

\section{Discussion}

Remarkably, the coronal outflow model fits the data in a small region of the parameter space, which allowed us to derive significant constraints on the active bundle of magnetic field lines ( $\mathrm{j}$ bundle). In particular, for 4U 0142+61 and 1RXS J1708-4009 the opening angle of the j-bundle $\theta_{j} \sim 0.1-0.2$, and its total luminosity (most of which is emitted in the $\mathrm{MeV}$ band, outside the observed spectral range) is $\sim 4 \times 10^{36} \mathrm{erg} \mathrm{s}^{-1}$. Both parameters are in line with theoretical expectations (Beloborodov 2009). Using Equation (3) one can estimate the product of the twist angle $\psi$ and the discharge voltage $\Phi$ in the j-bundle. We found $\psi \Phi \gtrsim(2-3) \times 10^{10} \mathrm{~V}$, consistent with $\Phi \gtrsim 10^{10} \mathrm{~V} 1$ This voltage is higher (at least by a factor of a few) compared with theoretical estimates of Beloborodov \& Thompson (2007) or the voltage inferred from the outburst decay in XTE J1810-197 (Beloborodov 2009).

The j-bundle parameters obtained for 4U 0142+61 and 1RXS J1708-4009 are comparable,

\footnotetext{
${ }^{1}$ The twist angle $\psi \gtrsim 1$ is expected for an active magnetar. The growth of $\psi$ beyond a critical value $\sim 3$ is prohibited by a global instability - the over-twisted magnetopshere inflates, forms an unstable current sheet, and ejects magnetic plasmoids, reducing the twist energy (Parfrey et al. 2013).
} 
1E $1841-045$

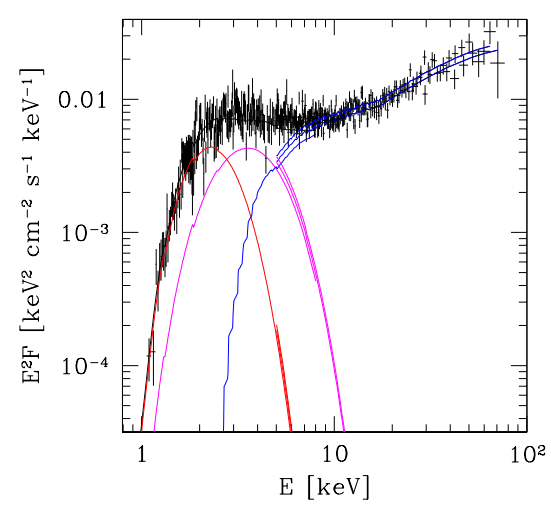

$4 \mathrm{U} 0142+61$

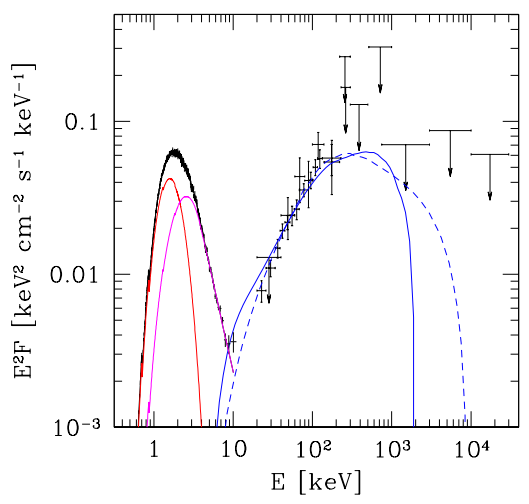

1RXS J1708-4009

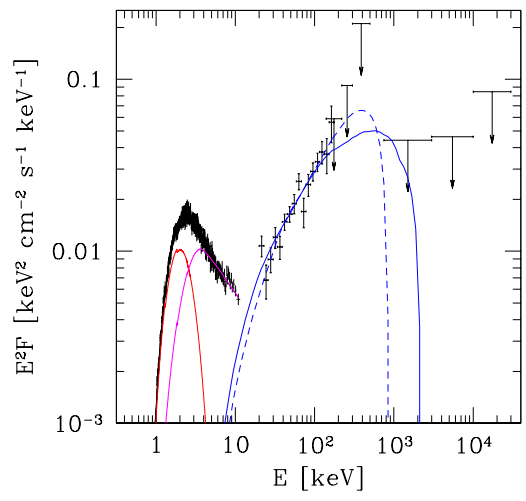

Fig. 3.- Best fits for the X-ray spectra of 1E 1841-045, 4U 0142+61, and 1RXS J1708-4009. Three spectral components are shown in each panel: the cold blackbody (red), the hot (modified) blackbody (magenta), and the coronal outflow emission (blue). For 4U 0142+61 and 1RXS J17084009, the dashed blue curve shows the best fit of the hard component obtained when only the phase-averaged spectrum is considered and the phase-resolved data are neglected. The data and models in the figure are not corrected for interstellar absorption. Only Swift XRT data are shown for 1E 1841-045 to avoid confusion in the figure (the actual analysis presented in An et al. (2013) includes Chandra and XMM data); the model curves split in two because of the offset in the normalization between the XRT and the two modules of NuSTAR.

which may be expected, as their spectra are similar. The spectrum of 1E 1841-045 is significantly different: the spectral index of its hard X-ray component is larger and the dip between the soft and hard components is smaller. We find that this difference is mainly explained by different angles $\alpha_{\text {mag }}$ and $\beta_{\text {obs }}$; the parameters of the j-bundle itself $\left(L_{j}\right.$ and $\left.\theta_{j}\right)$ are comparable for all three objects. The fact that two out of three magnetars appear to have a small angle between the magnetic and rotation axes $\alpha_{\text {mag }} \sim 0.1$ suggests that rotation plays a role in shaping the magnetic moment of the neutron star. Future measurements of phase-resolved spectra for other magnetars will allow one to

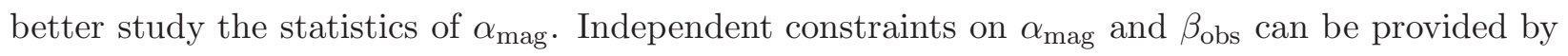
measurements of X-ray polarization by future missions (such as Astro-H, Takahashi et al. 2012).

The analysis of the hard X-ray component gives the magnetic flux in the j-bundle from which one can estimate its footprint area $\mathcal{A}_{j}$. The exact area and location of the footprint depend on details of the magnetic field near the star; a rough estimate for $\mathcal{A}_{j}$ may be obtained assuming a dipole field. A hot spot with area $\sim \mathcal{A}_{j}$ may be expected on the star. The obtained fits of the soft X-ray component are indeed consistent with the presence of a hot spot and give an independent estimate for its area $\mathcal{A}_{2}$. In 1RXS J1708-4009 and 1E 1841-045, $\mathcal{A}_{2}$ is consistent with $\mathcal{A}_{j}$, providing further support to our model. For $4 \mathrm{U} 0142+61$ we found $\mathcal{A}_{2} \approx 6 \mathcal{A}_{j}$, suggesting a hot spot extending around the $\mathrm{j}$-bundle footprint or indicating that the centered dipole configuration is a poor approximation to the magnetosphere near the star. 
Instead of studying the phase-resolved spectra in three phase bins, one could use coarse spectral binning and analyze more detailed pulse profiles. We did not attempt to fit the detailed pulse profiles, because they are significantly affected by moderate deviations from axisymmetry 2 Relaxing the axisymmetric assumption would make the model more flexible and difficult to describe using a small number of parameters. This would require a different, more complicated fitting procedure. Note also that the pulsed fractions of the three magnetars are moderate even at high energies, in the range of $30-40 \%$ at $\sim 100 \mathrm{keV}$ (W. Hermsen, private communication). This is consistent with approximate axisymmetry of the j-bundle, explaining why our simple model gives reasonable fits to the phase-resolved spectra in wide phase bins.

The coronal outflow model predicts that most of the luminosity is emitted in the MeV band. Future detectors with sensitivity better than that of Comptel would help to better constrain the location of the $\mathrm{j}$-bundle and the angle between the magnetic and rotation axes.

This work was supported by NASA ATP grant NNX 13AI34G.

\section{REFERENCES}

An, H., Hascoët, R., Kaspi, V. M., et al. 2013, ApJ, 779, 163

Beloborodov, A. M. 2009, ApJ, 703, 1044

-. 2013a, ApJ, 777, 114

—. 2013b, ApJ, 762, 13

Beloborodov, A. M., \& Thompson, C. 2007, ApJ, 657, 967

den Hartog, P. R., Hermsen, W., Kuiper, L., et al. 2006, A\&A, 451, 587

den Hartog, P. R., Kuiper, L., \& Hermsen, W. 2008a, A\&A, 489, 263

den Hartog, P. R., Kuiper, L., Hermsen, W., et al. 2008b, A\&A, 489, 245

Dib, R., Kaspi, V. M., \& Gavriil, F. P. 2007, ApJ, 666, 1152

—. 2008, ApJ, 673, 1044

Duncan, R. C., \& Thompson, C. 1992, ApJ, 392, L9

Durant, M., \& van Kerkwijk, M. H. 2006, ApJ, 650, 1070

\footnotetext{
2 The j-bundle emission is relativistically beamed along the magnetic field lines, especially in the hard X-ray band. Breaking the axial symmetry (concentration of electric currents in a range of magnetic longitude) affects the observed pulse profiles.
} 
Enoto, T., Nakazawa, K., Makishima, K., et al. 2010, ApJ, 722, L162

Gotthelf, E. V., \& Halpern, J. P. 2007, Ap\&SS, 308, 79

Ho, W. C. G., \& Lai, D. 2001, MNRAS, 327, 1081

Kuiper, L., Hermsen, W., den Hartog, P. R., \& Collmar, W. 2006, ApJ, 645, 556

Özel, F. 2001, ApJ, 563, 276

Parfrey, K., Beloborodov, A. M., \& Hui, L. 2013, ApJ, 774, 92

Rea, N., Zane, S., Turolla, R., Lyutikov, M., \& Götz, D. 2008, ApJ, 686, 1245

Takahashi, T., Mitsuda, K., Kelley, R., et al. 2012, in Society of Photo-Optical Instrumentation Engineers (SPIE) Conference Series, Vol. 8443

Thompson, C., \& Duncan, R. C. 1996, ApJ, 473, 322

Thompson, C., Lyutikov, M., \& Kulkarni, S. R. 2002, ApJ, 574, 332

Tian, W. W., \& Leahy, D. A. 2008, ApJ, 677, 292

This preprint was prepared with the AAS IATEX macros v5.2. 